Les acteurs locaux, les industriels, les décideurs se trouvent nécessairement au carrefour de la science et de la société, contraints de faire face à une complexité scientifique de plus en plus grande, à un débordement des frontières du local vers le global mais aussi à un système démocratique qui met en jeu un autre acteur : le public. Le Club CRIN-Environnement a organisé en 1995 avec le ministère de l'Environnement et l'Association Natures Sciences Sociétés-Dialogues* un colloque sur ce thème dont nous vous présentons ici quelques extraits.

\section{EXPOSÉ INTRODUCTIF}

Pourquoi le Club CRIN a-t-il choisi ce thème? Le ministère de l'Environnement avait organisé il y a quelques années à Arc et Senans, un colloque extrêmement intéressant intitulé : "Les experts sont formels ?", titre un peu ironique et avec un point d'interrogation mais ce colloque sur l'incertitude et l'expertise avait montré l'importance du sujet et soulevait nombre de réflexions fort judicieuses, encore d'actualité, sur l'incertitude et ce qu'on pouvait attendre de l'expertise.

Depuis lors, il y a eu des évolutions très importantes : la science évolue de façon continue, par contre le droit, la réglementation, le contexte international progressent par ruptures et connaissent une véritable mutation ; la sensibilité de la société s'est manifestement accentuée par rapport aux fractures diverses et aux catastrophes naturelles ; enfin, les entreprises, elles aussi, ont beaucoup évolué en mettant en place des risk-managements et des services de l'environnement qui n'existaient pas il y a cinq ans. Comme vous le savez, l'objectif des clubs CRIN est de faire dialoguer et travailler ensemble les acteurs économiques, entre. prises, associations, services de collectivités locales, etc., avec les milieux scientifiques et l'administration. Le point de départ qui nous permettra d'entrer ici dans le vif du sujet se résume en cinq postulats :

$1^{\text {er }}$ postulat sur lequel je crois qu'il n'est pas tellement utile d'insister devant cette audience : l'incertitude scientifique est omniprésente dans les décisions concernant l'environnement. Elle provient de l'état des milieux naturels qu'on ne pénètre pas facilement, des mesures et de leur variabilité, qui est fort grande lorsqu'il s'agit de teneur infime ou d'échantillonnage complexes, d'effets d'échelle dans le temps et dans l'espace, de réactions biologiques en chaine, de mesures d'impacts et de problèmes d'effets de seuil aux faibles doses, de comportements, de contre-stratégies qui se sont affinées et s'affinent sans cesse devant cette situation.

$2^{e}$ postulat : l'environnement est particulièrement cause du développement de cette incertitude. Pourquoi ? Parce que jusqu'à présent il existait des domaines traditionnels pour chaque acteur, qui en délimitant les territoires, aidaient les entreprises, les individus, les agents divers, les experts, à composer avec l'incertitude, en se cantonnant dans un domaine. Par exemple, pour un industriel, c'était sa production, l'intérieur du périmètre de ses installations qui lui permettaient d'ignorer l'extérieur; de cette façon, il avait au moins l'impression d'accéder à une maîtrise suffisante de ses produits, de ses problèmes, de ses risques d'accidents, etc. Le XIX ${ }^{\mathrm{e}}$ siècle a vu l'émergence d'un certain nombre de questionnements, par exemple sur les risques d'accidents ou risques tout court, mais c'était toujours à l'intérieur d'un périmètre étroit.

L'environnement a clairement fait éclater cette situation de cloisonnement, en obligeant les acteurs à s'intéresser et à se sentir concernés ou responsables en dehors de leur cercle interne mais aussi, et cela a été la
- L'Association NSS-Dialogues avait organisé ses Journées 1994 sur un thème un peu analogue qui était celui du Principe de Précaution. Une sélection des meilleures interventions sera publiée par I'INRA sous la direction scientifique d'Olivier Godard, en 1997. S'adresser à NSS-Dialogues, Bât. G, Université de Paris X, 200 avenue de la République, 92001 Nanterre cedex.

Tél. : (33) 0140977116. 
seconde phase de l'extension de l'incertitude par l'environnement, en étendant presque indéfiniment les frontières de ce qui était le cercle externe. On est passé à l'échelle globale, planétaire des impacts techniques et scientifiques et à la prise de conscience du phénomène d'inter-génération avec le développement durable. Ce qui a entrainé une amplification considérable de l'incertitude.

$3^{e}$ postulat : les scientifiques composent avec l'incertitude et même en tirent une dynamique d'action. Alors, là aussi ce n'est pas à l'assistance que j'apprendrais que, de tous temps, depuis des siècles, les scientifiques ont travaillé avec le doute et l'incertitude : tous les systèmes de calcul d'erreur, l'utilisation des lois statistiques en thermodynamique il y a un siècle, la théorie quantique plus tard, les lois de l'hérédité, la théorie des jeux, la théorie de l'information après la guerre, l'analyse systémique et, plus récemment, le développement de la théorie fond, tous ces outils scientifiques sont des outils de travail pour "faire avec" l'incertitude et ont donné lieu à des développements et des progrès scientifiques importants. On peut donc dire que le milieu scientifique travaille sur la base de controverses et se nourrit d'un développement de questionnements et d'incertitudes. Phénomène déjà soulevé par Bruno Latour et l'école de la sociologie de la science.

Par conséquent, il ne faut pas attendre des scientifiques qu'ils referment l'incertitude. Je crois qu'aujourd'hui, dans cette balance entre incertitude et certitude, la science est plutôt du côté d'un bascule. ment vers un développement de l'incertitude mais "contrôlée" dans un processus.

$4^{e}$ postulat : les agents économiques et hiérarchiques, c'est-à-dire les magistrats, l'administration, l'ensemble des agents non scientifiques qui appliquent les réglementations, sont eux, au contraire, mis dans une situation de malaise et d'inconfort par rapport à l'incertitude environnementale. C'est ainsi que les juristes travaillaient jusqu'à présent sur des certitudes que l'on demandait

à "l'expert". À partir du moment où celuici ne produit plus de la certitude, tout le système se trouve mis en question.

$5^{e}$ postulat : notre mode mental français a besoin d'être adapté, car il est particulièrement mal préparé à cette extension considérable de l'incertitude ; il y a en France une pratique cartésienne, autoritaire, centralisée, descendante, qui est opposée à la pratique beaucoup plus pragmatique d'autres pays ; nous avons certainement un ajustement plus difficile de ce fait.

En résumé, si vous ne contestez pas ces cinq postulats, nous pouvons entrer de plein pied dans le débat, nous demander comment agir dans cette situation, comment aider les agents économiques et d'autorité, les agents de la société civile, à prendre des décisions dans une telle situation. Tel est l'objet de cette journée.

Paul-Henri Bourrelier, (Président du Club CRIN-Environnement)

\section{L'APPROCHE D'UN RESPONSABLE LOCAL}

Je travaille dans une direction régionale de l'Industrie, de la Recherche et de l'Environnement, et Paul-Henri Bourrelier m'a demandé de vous présenter le témoignage, je ne dirais pas d'un homme de terrain, nous sommes tous hommes ou femmes de terrain, mais d'un responsable local. C'est donc dans ce sens que mon témoignage sera bâti. Je ne parlerai pas de phénomènes planétaires, je n'ai pas de légitimité à m'exprimer là-dessus. Je vous présenterai le point de vue de quelqu'un qui est engagé, parmi d'autres, dans la recherche locale de solutions, non pas de compromis, le terme peut-être déplaisant, mais d'équilibre au milieu d'un champ de contradictions très fort, d'un champ d'incertitudes tout aussi important. J'essaierai également d'examiner quelles peuvent être les attentes des responsables locaux vis-à-vis des scientifiques ou des "experts".

Dans le domaine de la pollution atmosphérique, il y a vingt ans, nous nous préoccupions surtout des poussières, main- tenant nous en sommes à essayer de décortiquer les phénomènes de pollution photochimique en ville; dans le domaine de la pollution de l'eau, nous ne traitions que de la pollution oxydable de façon organique traditionnelle, maintenant nous traquons les micro-polluants ; pour les friches industrielles, nous nous contentions parfois de reverdir ou d'engazonner, maintenant nous tentons d'imaginer des technologies de détoxication.

On perçoit donc nettement un mouvement continu dans le spectre des préoc. cupations et des problèmes à traiter qui vont vers une complexité croissante. Je pense que sur le terrain de l'environnement ou plus généralement des risques, l'action publique en France a subi trois grands fac. teurs d'évolution :

$1^{\text {ère }}$ évolution : le passage progressif d'une logique de flux, de problèmes ou de pollutions à traiter, à une logique de stocks. C'est tout le débat de la résorption des pol. lutions historiques ou des accumulations de risques, ce qui revient à près au même.

$2^{e}$ évolution : une complexité de plus en plus grande des connaissances et des technologies à maîtriser.

$3^{e}$ évolution : l'aspiration à une vision globale des problèmes d'environnement ou même technologique. Le domaine technologique recherche un peu sa théorie de la grande unification parce qu'on a compris que le phénomène de pollution est global, qu'il ne sert à rien de dépolluer l'eau si l'on ne sait pas en aval, traiter les boues des stations d'épuration et donc nous allons sur des terrains où les équations sont de plus en plus nombreuses et complexes.

Quelles peuvent être les attentes des décideurs, compte tenu du principe de précaution qui nous fait obligation de réagir, même dans une situation de forte incertitude, et d'agir, bien entendu, le plus intelligemment possible? Je voudrais ici simplement citer quelques thèmes qui me paraissent importants.

Le premier sujet d'angoisse pour un décideur local, c'est le débat sur les faibles 
doses. Bien sûr, j'ai l'impression que je vais enfoncer une porte ouverte, mais actuellement nous constatons un emballement dans le stress des populations à propos des expositions chroniques aux faibles doses. Prenez, par exemple, la réaction de l'opinion publique sur les possibles effets des champs magnétiques des lignes électriques, ou de l'exposition faible aux rayonnements ionisants ; nous retrouvons là les mêmes éléments que dans la controverse sur le stockage des déchets radioactifs ou de faible activité.

Prenez aussi le domaine de la toxicologie, où il faut bien dire que jusqu'à présent, nous avons surtout mesuré la toxicologie aiguë et non pas les toxicologies diffuses chroniques et permanentes. Là, les responsables locaux sont le plus souvent désarmés pour apporter des réponses ou des explications.

Je vais vous donner un exemple. Nous avons été confrontés à un problème causé par une usine de pâte à papier, à l'aval de laquelle se trouvait une grande agglomération qui traitait son eau potable par le chlore. Il se trouvait que les sujets de cette papeterie avaient une certaine affinité avec ce produit et produisaient une molécule soupçonnée d'être cancérigène. Il fallait pousser, d'une part, la collectivité à renoncer au chlore, d'où des investissements, et l'industriel, d'autre part, à dépolluer davantage, d'où d'autres investissements.

La recommandation de l'OMS qui était à l'origine de ces décisions, a donc été exposée aux élus et aux industriels, ainsi que le raisonnement qui y conduisait et qui pouvait se traduire de la manière suivante : une personne qui buvait deux litres de cette eau pendant 50 ans de manière continue devait avoir moins d'une chance sur un million de contracter un cancer pouvant être imputé à cette toxicologie. Ce jour-là, les personnes que nous avions en face de nous avaient visiblement un doute sur la santé mentale de leurs interlocuteurs.

Nous touchons là un point important parce qu'il rejoint toutes les pulsions quasimillénaristes que nous rencontrons parfois dans l'opinion publique ainsi que le problème des normes ou des seuils (des chiffres derrière lesquels on peut se retrancher), et qui, à cause d'organismes publics tels que l'OMS ou l'Union Européenne pour ne citer qu'elles, connaissent un mouvement continu vers la baisse.

Je crois véritablement que les facteurs de sécurité qui sont retenus, les coefficients de sécurité, au-delà de l'incertitude, prennent en compte également un autre phénomène : l'angoisse des populations. $\mathrm{Si}$ nous n'arrivons pas à expliquer sa portée réelle, et je crois que là les chercheurs ont un travail important à faire, nous n'arriverons pas à contrecarrer ce mouvement de réduction des seuils. Il est inhérent à notre système, qui est un système démocratique, auquel certains pourraient préférer un despotisme éclairé, peut-être plus efficace pour eux, mais ce dernier n'est pas le régime dans lequel nous vivons, nous devons donc expliquer et convaincre.

Le deuxième point que je voudrais souligner est que dans le domaine de l'environnement, nous attendons beaucoup en matière d'amélioration des capacités de mesure, de caractérisation des phénomènes, de modélisation. Dans le domaine des technologies qui permettraient de corriger ou prévenir les pollutions ou risques, il me semble qu'il y a un territoire qui mériterait d'être davantage exploré, c'est celui des solutions techniques ou méthodes dites conservatoires ou réversibles. Pourquoi ? Parce que ce principe de précaution nous oblige à agir mais également à ménager l'avenir. C'est surtout à l'occasion du stockage des déchets radioactifs souterrains que la question de réversibilité a été mise sur la place publique, mais il existe bien d'autres domaines, comme celui du confinement d'une pollution d'un sol, où, parce que nous n'avons pas encore trouvé la bactérie qui permettrait de différer tel ou tel type d'hydrocarbure, nous utilisons une technique que nous espérons réversible, avec bien entendu un certain nombre d'effets pervers qui empêchent la généralisation de telles solutions, transitoires ou réversibles.

Troisième et dernier point : celui de l'interdisciplinarité. On en a beaucoup parlé. 
Je crois qu'il faut l'étendre aux sciences dites molles. Pourquoi ? Parce que, tout d'abord, nous sommes dans des domaines, qu'il s'agisse de pollution ou de risque, où il faut agir non pas seulement sur les équipements et les procédés, mais également sur le comportement du citoyen, du consommateur, ou de l'usager, et qu'ensuite nous nous sommes aperçus depuis un certain nombre d'années que le facteur humain était déterminant dans la maitrise des problèmes de pollution et dans celle des risques. Je crois que dans cette interdisciplinarité que l'on a périodiquement évoqué ce matin, il ne faut surtout pas oublier les sciences dites molles.

M. Caffet

(DRIRE - Rhòne-Alpes)

\section{LE POINT DE VUE D'UN INDUSTRIEL}

Je m'occupe d'environnement chez Elf Atochem et je vais essayer, à partir de problèmes concrets, ceux des CFC et de l'ozone stratosphérique, d'examiner quelles leçons nous pouvons en tirer.

Tout d'abord, on sait que les CFC ont été inventés pour répondre à un risque. C'est la division frigidaire de General Motors, qui en 1930 a demandé à ses centres de recherche de trouver une molécule permettant d'abolir le risque présenté par les réfrigérateurs ménagers fonctionnant au $\mathrm{SO}^{2}$ ou à l'ammoniaque. Les chercheurs ont proposé d'utiliser les CFC et leur emploi s'est développé considérablement, aux alentours de 1940 dans d'autres domaines : les aérosols, les mousses et les solvants par exemple. Le marché a littéralement explosé, il est passé de 500000 tonnes en 1940 à 1200000 tonnes mondiales en 1986. En voilà à peu près les données : le monde consommait en moyenne un $\mathrm{kg}$ de $\mathrm{CFC}$ par personne et par an $(0,08 \mathrm{~kg}$ pour les pays en voie de développement). Elf-Atochem était très puissant dans ce domaine puisque nous produisions entre 20 et $25 \%$ du marché mondial, donc plus de 1200000 tonnes.

À ce moment-là, il n'y avait ni danger, ni risque. Ces produits étaient merveilleux. Ils étaient purs $(99,98 \%)$, avec une extraordinairement faible toxicité, non inflammables, la merveille des merveilles et en plus, pas chers.

Comment est venu le choc? Comme souvent, par des méthodes analytiques, et c'est un dénommé Lovelock qui a mis au point, en 1970, la méthode d'analyse par chromatographie à capture d'électrons et a démontré que ces produits s'accumulaient dans l'atmosphère, ou du moins, qu'il y avait une croissance de leurs concentrations. Une courbe des concentrations (en partie par trillions), montrait l'influence absolument considérable sur l'environnement.

C'est alors, fait en soi déjà peu ordinaire, que les chimistes se sont regroupés pour essayer de connaître le devenir de ces CFC dans l'environnement. Vingt sociétés chimiques mondiales, japonaises, coréennes, européennes, américaines, se sont réunies et ont fondé un groupe de recherche doté d'un budget raisonnable, 2 millions de dollars par an. Elles ont conduit des recherches pluridisciplinaires parce que nous avions, dans ce domaine, un énorme problème. Nous avions affaire à des secteurs de l'environnement qui s'ignoraient et nous avions besoin d'une conjonction d'efforts dans tous les domaines pour analyser le devenir de nos produits dans l'environnement. Malheureusement, juste à ce moment, deux savants, Rowlan et Molina, ont émis une hypothèse générée par la controverse Concorde, qui a fait un "boum" dans la recherche atmosphérique en annonçant que " les CFC doivent être comme les $\mathrm{NOx}$ produits par le moteur du Concorde, des destructeurs d'ozone. " Et nous avons eu à faire face à la première hypothèse de destruction de nos molécules dans la haute atmosphère.

Un premier point que je soulignerai à ce stade, c'est qu'il faut que les industriels soient au cœur de la réflexion scientifique. Il faut être dans le système pour pouvoir en comprendre les dangers et pour les "croire". Les industriels, comme tout un chacun, rejettent $a$ priori les mauvaises nouvelles surtout lorsqu'elles viennent de l'extérieur et donc il vaut mieux avoir nos propres scientifiques parce que alors nous les admettons.
Qu'on fait alors les industriels? Ils ont subi les aléas de la science, qui ont été considérables. Un graphique pourrait essayer de montrer les potentiels de destruction de la couche d'ozone, imaginés au fil des années... En 1974 on parlait d'une baisse considérable d'ozone de $15 \%$, en 1976 ce chiffre était réduit à $7 \%$. Il est évident que les recherches effectuées pour trouver des produits de substitution, chez Elf-Atochem, comme chez nos concurrents, ont subi ces àcoups. Nous avons entrepris des études et ensuite nous nous sommes dits " ce n'est pas la peine " ; puis on a remis en route des équipes. On a fait des recherches de toxicité, les brevets bien sûr ont été pris, un peu d'application a été développé mais cependant, à ce stade, aucune production n'a été entreprise. Pourtant, un jour, les scientifiques sont venus avec un résultat absolu : l'ozone trand-panel qui démontrait qu'il y avait une baisse d'ozone significative. Â ce moment là tous les industriels se sont rués sur la mise en cuvre et la construction de nouvelles unités pour produire le $134 \mathrm{~A}$, produit qui tient en compte les problèmes de sécurité, d'environnement, de performance.

Le problème c'est que toutes les usines étaient construites mais que les clients eux, n'étaient pas là. De manière surprenante, les gens chargés de la réglementation, les industriels producteurs, les chimistes et les scientifiques avaient bien formé un petit groupe pour suivre l'évolution scientifique mais les industriels en aval, c'est-àdire nos clients directs, n'avaient pas été associés à cette démarche.

Ce sera là mon deuxième point : l'erreur de la chimie et peut-être aussi des pouvoirs publics, a été de rester dans un monde relativement étroit, de ne pas avoir associer tous les acteurs concernés. On s'est aperçu ensuite que le client final était certes bien informé, mais que lorsque on lui disait de payer le produit trois fois plus cher, il oubliait cette information. Entre un client qui déclare son intention d'acheter et un client qui achète, il y a l'écart du prix. Il est évident que la chimie n'a pas suffisamment communiqué. Et la science, comme tou- 
jours, a progressé et extrêmement rapidement.

Mais revenons à l'évolution un peu schématique de la chimie au travers de l'histoire de l'ozone stratosphérique : du calme plat des années soixante-dix en passant par le problème du Concorde pour arriver en 1988 au problème de trou d'ozone du Pôle que personne n'avait vu venir, sauf un savant anglais habitué du pôle, qui faisait cela de manière routinière et classique et qui a enfin publié après avoir vérifié plusieurs fois ses calculs.

La première réaction des Américains, de la NASA, a été de dire qu'il s'était probablement trompé, puisque leurs appareils sur le satellite TOMS (Total Ozone Mapping Systems), n'avaient rien décelé. Mais ils n'avaient rien vu car les Américains avaient mis un filtre sur les données en disant que toute donnée anormale serait écartée. Effectivement, la baisse d'ozone étant anormale, elle était écartée.

Ayant vérifié tout cela, on est tombé dans un peu de panique, puisque personne ne l'avait prévu. Alors on a eu une sorte d'hyper-réaction des réglementateurs. On a d'abord eu le Protocole de Montréal, avec un titre magnifique du journal Libération en première page : "Ozone espace protégé ". Vous noterez que c'était le 3 janvier 1989, une date où normalement il n'y a pas grand chose à dire probablement. Le Protocole de Montréal date de 1987, c'était donc la première année d'application.

Nous, chimistes, avions l'impres. sion d'être effectivement sauvés par ce protocole. Hélas, hélas, le problème du trou d'ozone, plus un certain nombre d'excitations réglementaires a provoqué une évolution faramineuse de l'accélération réglementaire. En 1987, le protocole de Montréal demandait simplement $50 \%$ de baisse dans la consommation du CFC. En 1990, trois ans après il préconisait l'arrêt total en l'an 2000. En 1992 le protocole a réclamé l'arrêt fin 1995 et immédiatement après l'Union Européenne l'a décrété pour fin 1994. En cinq ans, on est passé de $50 \%$ à $100 \%$ avec un horizon en l'an 2000 à un horizon beaucoup plus proche.
La première conséquence, c'est que les industriels qui avaient prévu dans le lancement de leurs produits de remplacement du CFC un timing relativement précis pour le pilotage, l'environnement, l'échelle industrielle, l'application, l'adaptation, l'extension des capacités, ont vu tout cela littéralement balayé. Nous avons eu à faire face à une accélération des décisions, en particulier des décisions d'investissement pour mettre un produit sur le marché sans avoir même pu terminer les études de toxicologie. Nous avons pris des risques financiers et industriels considérables. Je me rappelle une réunion de direction où le directeur général a demandé au toxicologue : «Vous avez le choix entre deux produits, A et B ; lequel choisissezvous? "Réponse : "Mais, Monsieur, nous n'avons pas fini les résultats de... "... " Je ne vous demande pas le point sur votre science, lequel choisissez-vous ? ". Il a choisi et les investissements sont partis sur quelque chose qui n'était pas finalisé. Cette accélération a fait prendre des risques considérables aux industriels.

Je crois que les conclusions qu'il faut tirer de cela sont les suivantes :

- Il faut être à l'intérieur du système, gérer au mieux les risques en les analysant. Dans ce cas, nous n'avons pas su gérer les risques perçus par notre aval, c'est-à-dire par les industriels clients et par les consommateurs. Il y a là un manque d'information ou de communication que la chimie et les pouvoirs publics n'ont pas fait à temps et correctement.

- Il y a un deuxième risque plus difficile mais du même type, c'est celui perçu par les médias et à travers eux par les politiques. Il ne sert à rien d'avoir développé des produits s'ils sont refusés par le public en vertu de sa perception différente d'un risque, peutêtre illogique, en tout cas pas dans notre logique.

L'investissement du chimiste doit tenir compte de sa propre réalité et aussi de la perception de cette réalité par ses clients.

M. Verhille (Elf-Atochem) 